\title{
STUDY ON MOSAIC AND UNIFORM COLOR METHOD OF SATELLITE IMAGE FUSION IN LARGE SREA
}

\author{
LiuShuhan $^{1, *}$, LiHongzhou $^{1}$, WangXia ${ }^{1}$, GuoLi $^{1}$, WangRui $^{1}$ \\ ${ }^{1}$ Satellite Surveying and Mapping Application Center, National Administration of Surveying, Mapping and Geo-information, \\ Beijing, P.R. China - liush@ sasmac.cn
}

KEY WORDS: Large area , Multi-temporal, Satellite image mosaic , Automatic uniform color

\begin{abstract}
:
Due to the improvement of satellite radiometric resolution and the color difference for multi-temporal satellite remote sensing images and the large amount of satellite image data, how to complete the mosaic and uniform color process of satellite images is always an important problem in image processing. First of all using the bundle uniform color method and least squares mosaic method of GXL and the dodging function, the uniform transition of color and brightness can be realized in large area and multi-temporal satellite images. Secondly, using Color Mapping software to color mosaic images of 16bit to mosaic images of 8bit based on uniform color method with low resolution reference images. At last, qualitative and quantitative analytical methods are used respectively to analyse and evaluate satellite image after mosaic and uniformity coloring. The test reflects the correlation of mosaic images before and after coloring is higher than $95 \%$ and image information entropy increases, texture features are enhanced which have been proved by calculation of quantitative indexes such as correlation coefficient and information entropy. Satellite image mosaic and color processing in large area has been well implemented.
\end{abstract}

\section{INTRODUCTION}

Since the beginning of the 21 st century, the ability of human to obtain the surface dynamic information of the earth has been further strengthened, and the technical ability of remote sensing data acquisition has been comprehensively improved (He G J, et al., 2015). In recent years, remote sensing data has been widely used in national economic construction, especially in large-scale resource environment survey. In the backdrop of the global environmental change, our country has carried out such as land use, resources, forest resources and ecological environment evaluation of projects, these projects development depend on national Mosaic, high spatial resolution remote sensing satellite data in (Guo H D, et al., 2014). In view of the area of China, Chinese academy of sciences institute of remote sensing and digital earth by using a series of Landsat satellite images of the received data, has released four period Mosaic products (RTU product data service, 2000, 2005, 2010 and 2014); The domestic satellite has the national Mosaic data made from the image of BJ-1(Wang A H, et al., 2009) and the CBERS - 1 satellite. Since 2010, China has launched a major special project on high-resolution earth observation system. The successive launches of the GF-1 and GF-2 satellites which provides rich and high quality data sources for Chinese and high spatial resolution satellite Mosaic products. The successful launch of the ZY3 mapping satellite that the long - term, continuous, stable and high - resolution stereo images and multi - spectral images are obtained. Li Deren verified that the uncontrollable positioning accuracy of ZY3 mapping satellite was better than that of $15 \mathrm{~m}$ (2012). With the control point elevation, the precision of the plane position is better than $3 \mathrm{~m}$ and $4 \mathrm{~m}$ respectively. The accuracy requirement of $1: 50,000$ scale map is satisfied (Liu G D, et al., 2015). Geometric accuracy verification test of ZY3 Mapping satellite data concluded that the precision of plane position and height difference is $3.2 \mathrm{~m}$ and $1.8 \mathrm{~m}$ based on which the accuracy of DSM and DOM are $2.07 \mathrm{~m}$ and $2.92 \mathrm{~m}$ is better than 1:50 000 topographic map specification (Tang $X$ M, et al., 2012). Therefore, the ZY3 mapping satellite could well serve the national geographic information 1:50, 000 mapping results and 1:25,000 and larger scale map updates. Therefore, to carry out the research on satellite image Mosaic technology for high-resolution satellite images and resources and land image mosaic product is not only of great scientific value, moreover, it is of great significance to establish the national resource environment remote sensing database and expand the application range of domestic satellite data (Long $\mathrm{T}$ F, et al., 2014).

\section{RESEARCH METHODS}

\subsection{Data source}

ZY3 surveying and mapping satellite is China's first self-developed series of civil three-dimensional surveying and mapping satellites, by stereoscopic observation, we can measure 1:50,000 scale topographic map and providing services for land, resources, agriculture, forestry and other fields. ZY3 fills the gap in the field of Chinese stereoscopic mapping.ZY301 is equipped with 4 cameras, one of them is $2.1 \mathrm{~m}$ ground resolution imaging machine, two $3.5 \mathrm{~m}$ resolution front view, rear video camera and one $5.8 \mathrm{~m}$ resolution multispectral imager. ZY302 is based on this and the resolution of the front and rear video cameras is respectively $3.5 \mathrm{~m}$ to $2.5 \mathrm{~m}$.

GF-1 satellite is the first star in the major special space-based system for the earth observation system. Its main purpose is to break through the optical remote sensing technology combining high spatial resolution, multispectral and high temporal resolution. Two $2 \mathrm{~m}$ spatial resolution full-color / $8 \mathrm{~m}$ spatial resolution multispectral cameras and four $16 \mathrm{~m}$ spatial resolution multispectral wide camera are configured.

GF-2 satellite is the first civilian optical remote sensing satellite developed in China with a spatial resolution better than 1 meter equipped with two high resolution $1 \mathrm{~m}$ full color and $4 \mathrm{~m}$ multispectral cameras. It has the characteristics of sub-meter 
space resolution, high positioning accuracy and quick attitude maneuver ability and has effectively improved the comprehensive observation efficiency of satellite and reached the international advanced level.

\subsection{Technical route}

In this paper is to correct the image as a starting point, the use of the software platform, auxiliary PS software of remote sensing image processing software, such as satellite image fusion, Mosaic pretreatment and edit, adjust the template, set the cutting such as image processing, finally get norm image products. The technical process is shown in figure 1 .

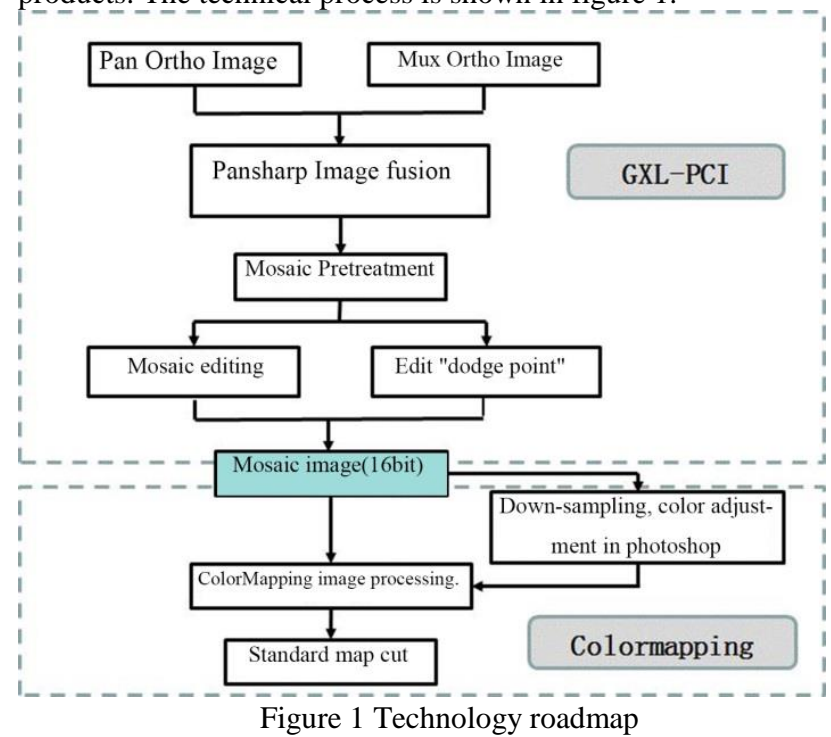

Firstly, the whole color and multispectral positive images were collected in the region, and pansharp fusion was carried out, and the waveband recombination (321 bands) was synchronized, and the true color fusion image was obtained. Second, using GXL software to pretreat fusion images, using the dodge point function of Mosaic tool software to adjust the color of adjacent images, and edit the automatically generated Mosaic line. According to certain features of the ground, the mosaic line is edited, and the mosaic image is obtained and the color of the image has been largely eliminated. Then the mosaic image is reduced to $20 \mathrm{~m}$, and the template is obtained. Adjust the template in Photoshop. Finally, using ColorMapping software, the Mosaic image was evenly matched with the color of the template. After cutting and cutting, we get the final standard parting shot image product.

GXL-PCI provides a variety of satellite image uniform color methods. One Bundle (Bundle) method by calculating each overlap with images "beam" method between average grey value of information, such as to adjust the image and the adjacent image gray value in order to reach a color balance which has good effect. In addition, GXL software provides the ability to artificially increase the dodge point. It can adjust the local color information of adjacent images and realize color equalization. Dodge points can be set to either side changes or side changes by artificially increasing the "dodge point", the image color of different seasons can be closer to achieve the best mosaic effect.

ColorMapping is a set of satellite image uniform color processing software for geo-coded reference images. By using the color mapping transform algorithm, the color matching between the image and the reference image can be processed. In order to reduce the loss of layers and the loss of saturation in the uniform color process, the data processing effect is improved. It has solved the information loss problem of the satellite image 16bit transferring 8bit and improved the efficiency of image processing.

\subsection{Research area}

The technical route of this article is carried out in a million units of national standard. The study area selected K50 which includes Beijing, Hebei, Liaoning and Inner Mongolia autonomous region. In this picture, various types of land cover, including vegetation, urban and rural areas, industrial and mining, residential land, water and sand, etc., provide a good research foundation for subsequent inlays and homogeneity.

\subsection{Uniform color evaluation index}

2.4.1 Correlation coefficient: The correlation coefficient is used to reflect the similarity between the image and the original image after fusion. The higher the correlation value, the higher the fidelity of the image spectrum and the better the fusion quality. The formula is:

$$
\mathrm{R}=\frac{\sum_{\mathrm{i}=1}^{\mathrm{m}} \sum_{\mathrm{j}=1}^{\mathrm{n}}\left[\mathrm{f}_{1}(\mathrm{i}, \mathrm{j})-\overline{\mathrm{A}_{1}}\right]\left[\mathrm{f}_{2}(\mathrm{i}, \mathrm{j})-\overline{\mathrm{A}_{2}}\right]}{\sqrt{\sum_{\mathrm{i}=1}^{\mathrm{m}} \sum_{\mathrm{j}=1}^{\mathrm{n}}\left[\mathrm{f}_{1}(\mathrm{i}, \mathrm{j})-\overline{\mathrm{A}_{1}}\right]^{2}\left[\mathrm{f}_{2}(\mathrm{i}, \mathrm{j})-\overline{\mathrm{A}_{2}}\right]^{2}}}
$$

In the formula, $f_{1}(i, j)$ is the image pixel value of the uniform color front template, $f_{2}(i, j)$ is ColorMapping after uniform color image pixel value, $\overline{\mathrm{A}_{1}}$ and $\overline{\mathrm{A}_{2}}$ represent the pixel values of the two images respectively.

The larger the $\mathrm{R}$ value, the greater the correlation between the uniform color image and the pre-uniform image. The more similar the color is, the better the automatic uniform color effects.

2.4.2 Information entropy: Image information is the quality of fusion image from the Angle of pixel probability. Its theoretical basis is information theory, and the common index has information entropy. Information entropy is considered from the statistical characteristics of the whole information source. It is a measure of the degree of disorder in the material, and the image entropy is often used to measure the image information. By analysing the information entropy of the image, the detailed performance of the image can be obtained, and the greater the entropy value, the richer the information. According to Shannon information theory principle, for an image, the information entropy calculation formula is:

$$
H=-\sum_{i=0}^{L} P_{i} \log _{2} p_{i}
$$

In the formula, $\mathrm{L}$ is the gray scale, which is the probability density of pixel of the single-band image pixel value of $i$.

\section{RESULTS 3}

\subsection{Time efficiency analysis}

With the acceleration of the hardware and software environment, how to make faster, more efficient and less human participation methods and technologies have been gradually applied to satellite image data. Since the computer can work continuously, how to save the labor participation time and cost is required to be calculated and calculated. Here's an example of each of the 
millions of images. The statistical utilization of satellite images of $\mathrm{ZY} 3$ was used to inlay the time and human participation of each process.

\begin{tabular}{|lll|}
\hline & Way to work & Time statistics \\
\hline Data preparation & manual & - \\
Ortho-rectification & automatic & $10 \mathrm{~min} / \mathrm{scene}$ \\
Image fusion & automatic & $5 \mathrm{~min} / \mathrm{scene}$ \\
Mosaic pretreatment & automatic & $2 \mathrm{~h}$ \\
Mosaic line editor & manual & $3 \mathrm{~d} / \mathrm{million}$ map \\
Image mosaic & automatic & $10 \mathrm{~h}$ \\
Reference image & manual & $4 \mathrm{~h} / \mathrm{million}$ map \\
ColorMapping & automatic & $10 \mathrm{~h} / \mathrm{million}$ map \\
Image cut & automatic & $3 \mathrm{~min} / \mathrm{map}$ \\
\hline
\end{tabular}

Table 1 time statistics of workflow

From the statistical results of the table above, in addition to the time for data preparation, the following artificial participation links are mainly mosaic editing and reference images. A total of 3.5 working days were required to complete the image Mosaic work of some $300,000 \mathrm{~km}^{2}$.

\subsection{Analysis of mosaic results}

Figure 2 is the Mosaic uniform color process of ZY3 satellite image of K50. Figure (a) is the fusion image. Figure (b) is the adjustment of edge effect after GXL-PCI software, and the adjacent images are uniformly over. Figure (c) is to reduce the result of figure (b) to $20 \mathrm{~m}$, and the image size is reduced to 3-4GB. Through reference image formed after artificial color mixing, the output of ColorMapping software is used.
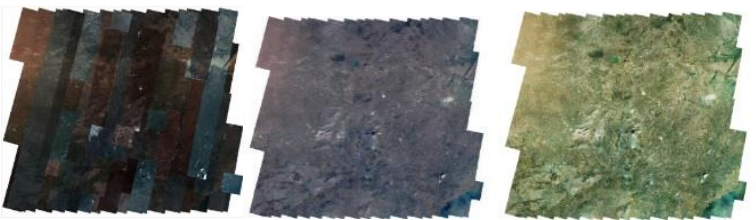

Figure 2 Mosaic uniform color process of K50

The above image reflects the above process operation. In this way, the color uniformity between adjacent images is achieved, and the effect of artificial color mixing is realized, and the work load is reduced.

Evasion point refers to the adjacent image mosaic line between random increase points. Realize the local scope of image color consistency through statistical partial overlap area image histogram equalization processing. In the region where the image season is different, the number and density of dodge points can be increased appropriately to achieve uniform color transition between images. In addition, the dodge point can be set to either side changes or side changes, which can be applied to the case where there is a cloud cover on one side.
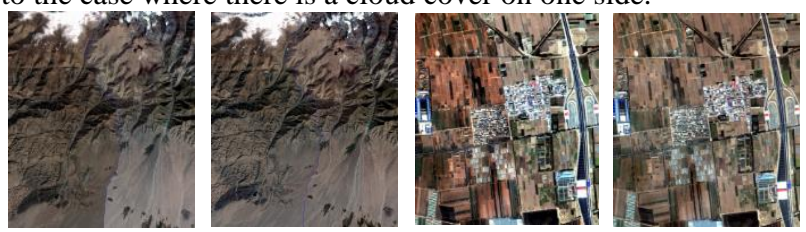

Figure 3 comparison of the dodge point edition

Figure 3 respectively reflect the mountain area and urban area and original color difference between image and evasion point after the adjustment effect, mountainous image acquisition time respectively on October 1, 2016 and 2016 on August 5, left and right image brightness contrast is bigger. After adding 3 dodge points, the color and brightness of the left and right images are even excessive. From the visual effect, there is no discernible trace. The two adjacent images in the urban area, the acquisition time is November 26, 2016 and April 18, 2017, respectively. By adding 5 dodge points, the color between the city and the plot reached an even excess.

In addition, the use of " dodge points " edit function can also be the image grey value such as Marine, desert regions were uniform color processing, at the same time, the four seasons in the north of the city, summer image contrast is very big, also can use dodge points method for editing.

Setting can effectively evade realize evenly between adjacent image color, and texture of image details loss is not big, through evasion point before and after the adjustment of histogram can be seen that image mean to change, and histogram curve change is very small.

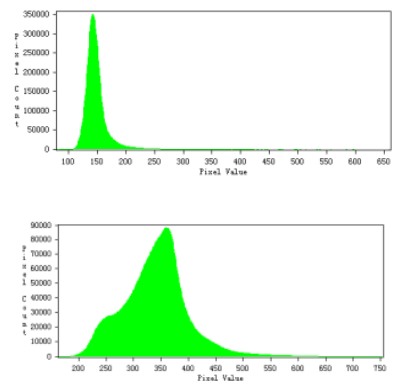

(a)
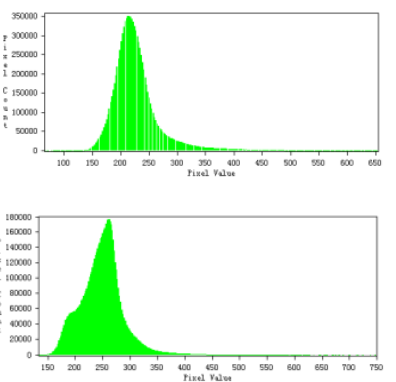

(b)
Figure 4 (a) adjacent image histogram before the adjustment

(b) adjacent image histogram after the adjustment

Above reflect the changes of the urban area before and after the adjustment histogram, figure 3 before the adjustment of image gray scale average of 148 and 362 respectively, difference is very big, the adjusted image gray scale average of 219 and 238 , gap decreased significantly.

\subsection{Analysis of uniform color results}

The working principle of ColorMapping software is based on the reference image. The color adjustment of the embedded image is made and the image drop is completed synchronously which 16bit is reduced to 8bit. The most intuitive way to evaluate the color mixing effect is to judge the effect of color mixing by looking at the texture details and tone of the images before and after the information of the mountain and urban areas. In addition, two quantitative indexes can be used to judge the effect of software toning: One is the image information entropy. Information entropy is an index reflecting the detail characteristics of image texture, and the larger the value is, the more information is available. Second, the correlation coefficient between image and reference image after color adjustment is to judge the color deviation of the image before and after the color adjustment by calculating the correlation between the two images.

In this paper, 5 Standard 100,000 maps of K50 are statistically analyzed and all the categories of objects are included. The results are shown in table 1 . The correlation coefficient of each band is greater than $95 \%$ between reference image and uniformed image. The correlation between uniform color image and reference image is high and the color of uniform color 
image color is guaranteed. After uniformed, the information entropy of each band is increased, the texture information after uniform color is enhanced and more abundant and the uniform color image has achieved good results.

\begin{tabular}{|l|c|l|l|l|l|l|}
\hline & band & Zone1 & Zone2 & Zone3 & Zone4 & Zone5 \\
\hline (a) & 1 & 5.77 & 5.16 & 4.75 & 4.88 & 6.25 \\
& 2 & 5.51 & 5.42 & 4.62 & 5.12 & 5.88 \\
& 3 & 5.34 & 5.41 & 4.51 & 4.76 & 5.81 \\
\hline (b) & 1 & 6.11 & 6.90 & 5.07 & 5.62 & 6.80 \\
& 2 & 5.71 & 6.85 & 5.05 & 5.69 & 6.74 \\
& 3 & 5.48 & 6.46 & 5.02 & 5.23 & 6.72 \\
\hline (c) & 1 & 0.973 & 0.976 & 0.967 & 0.988 & 0.958 \\
& 2 & 0.961 & 0.985 & 0.982 & 0.986 & 0.977 \\
& 3 & 0.074 & 0.962 & 0.977 & 0.991 & 0.965 \\
\hline
\end{tabular}

Table 2 quantitative statistical results of uniform color quality (a) Image information entropy before uniform color (b) Image information entropy after uniform color (c) Correlation coefficient between reference image and uniform color image

\section{CONCLUSION}

This paper use the "dodge point" function provided by GXL software and the automatic uniform color function of ColorMapping software. Brightness and color of adjacent images can be adjusted locally. The color balance of global image can be realized quickly by using the semi-automatic method. At the same time, it is of reference value to convert the 16bit image to 8 bit based on the reference image matching method. In this paper, visual interpretation and quantitative statistics are used in the evaluation of uniform color quality. The experiment reflects that the "dodge point" function can realize the uniform color transition of the image, and the visual effect is better. ColorMapping's uniform color process retains the image color and texture feature information completely. Through correlation coefficient and information entropy quantitative index calculation, it is proved that the correlation of mosaic before and after uniform color is over 95\%. Image information entropy increases, texture features are enhanced, large area satellite image Mosaic and uniform color processing are well realized.

\section{ACKNOWLEDGEMENTS}

This work was supported by the High Resolution Images Surveying and Mapping Application System of China (Project No. AH1601-11)

\section{REFERENCES}

He G J, Wang L Z, Ma Y, et al., 2015. Processing of earth observation big data: Challenges and countermeasures. Chinese Science Bulletin, 60( 5 /6) : 470-478.

Guo H D, Fu W X, Li X W, et al., 2014. Research on global change scientific satellites. Science China Earth Sciences, 57 (2) :204-215.

The plan of RTU product data service [EB/OL] . (2015-03-10) [2016-06-16] . http: / /ids. ceode. ac. .cn /rtu /index.aspx.

Wang A H, Chi Y B, Wang Z Y et al. 2009. Study on mosaic technology and mapping of China based on the multispectral images of Beijing - 1small satellite. Journal of Remote Sensing, 13( 1): $83-90$.

Wei Z, Chen Z C, Zhang B, et al. CBERS-1 digital images mosaic and mapping of China. Journal of Image and Graphics, 2006, 11(6): $787-791$.

Li Deren. 2012. China's first civil tri linear array stereo mapping satellite-ZY3 mapping satellite. Journal of surveying and mapping, 41(3) : 317-322.

Tang X M, Zhang G, Zhu X Y, etc. 2012. Preliminary verification of the construction and accuracy of the three-line array imaging geometry model of ZY3 mapping satellite. Journal of surveying and mapping, 41(2) : 191-198.

Jiang Y H, Zhang G, Tang X M, etc. 2013. High precision geometry calibration of ZY3 mapping satellite trilinear array image. [J].Journal of surveying and mapping, 42 (4) :523-529.

Cheng Q, Chen J F. 2015. Research on the extraction method of land cover information in southern coastal land of Hangzhou Bay based on GF-1 Image. Journal of Natural Resources, 30 (2) : 350-360.

Liu G D, Wu M Q, Niu Z, et al. 2015. Investigation method for crop area using remote sensing sampling based on GF-1 satellite data. Transactions of the Chinese Society of Agricultural Engineering, 31( 5) : 160-166.

Zhao S H, Wang Q, Yang Y P, et al. 2015. The demonstration research of GF-1 satellite data monitoring environment application. Satellite Application, 2015( 3) : 37-40.

Sun J B.2013. Principles and Applications of Remote Sensing [M]. 3rded. Wuhan: Wuhan University Press,2013:105-116.

Long T F, Jiao W L, He G J. 2014. Nested regression based optimal selection of rational polynomial coefficients. Photogrammetric Engineering and Remote Sensing, 80( 3) : 261-269. 